\title{
Knowledge management and decision support for electrical power utilities
}

\author{
G.M. West ${ }^{1}$, S. M. Strachan ${ }^{1}$, A. Moyes ${ }^{1}$, J.R. McDonald ${ }^{1}$, B. Gwyn ${ }^{2}$ and J. Farrell ${ }^{3}$ \\ ${ }^{1}$ Department of Electrical and Electronic Engineering, University of Strathclyde, UK \\ ${ }^{2}$ National Grid Company, UK \\ ${ }^{3}$ ScottishPower, UK
}

\begin{abstract}
This paper describes a case study for a knowledge management project carried out in conjunction with two major UK electrical utilities. The focus of the work is on the design of protection schemes for electrical power transmission systems. A brief overview is provided of the traditional protection system design processes implemented within both companies and the associated shortcomings relating to the management of the data, information and knowledge throughout this process. The development of the Design Engineering Knowledge Application System (DEKAS), designed to address the knowledge management issues within both companies, is described, and the perceived benefits this system offers the existing protection design and application process of each company will be discussed.
\end{abstract}

\section{Introduction}

What actually constitutes 'knowledge'? and what are the distinctions between 'knowledge', 'data' and 'information'? are frequently asked questions.

The general consensus of accepted definitions for each of these entities are (Schreiber et al., 1999):

- Data - Uninterpreted raw material providing the basis for analysis.

- Information - A collection of data interpreted to provide meaning.

- Knowledge - The collective body of data, information and reasoning which provides a sense of purpose relating to a particular task.

While the definitions of data, information and knowledge may be open to conjecture, the close relationship which exists between each of these entities and the critical role each performs in the day-to-day function of every organization is clearly apparent. Knowledge management concentrates on optimizing the utilization of knowledge within organizations, supporting fast, high-quality decision making and ultimately delivering increased productivity and tangible financial benefits. The retention and sharing of knowledge can also be supported through the implementation of knowledge management within an organization.

Knowledge engineering methods were initially developed for the design of knowledgebased systems (KBS). The application of knowledge engineering techniques have diversified into related areas such as requirements engineering and knowledge management. Knowledge engineering has a significant role to play in the management of knowledge as a resource within organizations. Through the application of knowledge engineering techniques, existing organizational processes and procedures can be appreciated and captured, enabling streamlining and optimization of existing processes 
and knowledge resources. Knowledge management within an organization can also receive support in the form of knowledge-based systems, often requiring a more detailed knowledge engineering approach than that required for process modelling.

This paper considers the application of knowledge engineering for the purposes of process modelling and the design of knowledge-based support, satisfying the knowledge management requirements of the existing protection design and application processes within two leading UK utility organizations.

\section{DESCRIPTION OF DOMAIN}

\section{Protection of the electrical transmission system}

Electricity is delivered from generators (power stations), throughout the country at high voltage, via a transmission grid consisting of a network of overhead lines and underground cables. Where concentrations of electrical load exist, the voltage level is stepped down and delivered to consumers through a distribution network. Both transmission and distribution networks must be protected against faults and unforeseen circumstances. In response to a fault event on the network it is essential for the fault to be removed from the network as quickly as possible, in order to minimize the risk of damage to connected plant and injury to the public. Fault clearance can be achieved via a protection scheme consisting of a combination of switchgear and relays (primary and secondary protection) which will detect and isolate faults on the network (Wright and Christopoulus, 1993). The transmission network is composed of large numbers of expensive plant items and a fault on the transmission network may also lead to a widespread outage affecting thousands of customers over a large geographic area. It is for these reasons that protection schemes associated with transmission networks are generally more complex than those associated with distribution networks. This paper considers the existing process implemented during the design and application of protection schemes for electrical transmission systems and addresses the knowledge management issues associated with this process.

\section{Protection system design}

The task of designing a protection scheme associated with the electrical power system is complex, involving consideration of multiple constraints. Typical constraints arise from primary transmission plant layout, interfacing with existing protection schemes on adjacent parts of the system, safety, reliability and cost. The protection scheme design process represents a compromise between these different, and often conflicting, factors.

Traditionally, the design of protection schemes has relied heavily upon the knowledge and experience of the utility protection engineers. When designing a protection scheme, the engineer usually refers to similar projects in which they have been previously involved. The design of a protection scheme utilizes this experience, combined with access to relevant company documentation, systems information and other data/information resources. 


\section{SHORTCOMINGS OF EXISTING PROCESSES AND THE ASSOCIATED KNOWLEDGE MANAGEMENT ISSUES}

The main shortcomings and knowledge management issues relating to the management and dissemination of data, information and knowledge within the existing design processes of each company have been identified as follows:

- A protection engineer must draw upon resources contained within a variety of formats combined with their own 'know-how' and experience. At present searching for, and gathering of relevant documentation for a particular design project involves a considerable number of man-hours.

- The company standards and guideline documents are updated as required to reflect changes to company policy. A more effective way of disseminating the information throughout the company is required. It may be more practical to constantly remind design engineers of changes to company policies and documentation as and when required through out the duration of a design project, rather than only when the initial change occurs.

- Protection engineers, and in particular less experienced engineers, may be unaware of the existence of potentially useful documents and/or their location within the company file structure.

- Protection engineers often refer to their own experience of previous protection scheme projects in an attempt to establish similarities between past and present designs, leading to re-use of design knowledge. Capturing this tacit knowledge from experienced protection engineers enables experiential knowledge to be shared across the company, supporting a more consistent approach to the protection scheme design and application process.

- There is a desired intention to increase the use of existing experiential knowledge within the company in the training of new engineers.

- Another barrier to successful dissemination of experiential knowledge is physical distance, where engineers are based throughout the country.

- There is a requirement to automate existing manual processes within the overall protection design and application process in an attempt to increase productivity.

- Although guidelines relating to the various activities within the design process are documented within company procedures and standards, individual engineers may employ different approaches to the completion of a particular task. There exists a requirement to standardize the approaches taken by protection engineers to the implementation of the design process activities through the promotion of best practices.

\section{DEVELOPMENT OF THE DESIGN ENGINEERING KNOWLEDGE APPLICATION SYSTEM (DEKAS)}

Following the identification of the existing process shortcomings, the next stage of the project aimed to enhance the protection design and application process by alleviating these shortcomings and where possible eliminating them completely.

The enhancement of the existing process through the introduction of a knowledge management philosophy led to the development of the Design Engineering Knowledge 
Application System (DEKAS). DEKAS provides engineering support during the protection design and application process in the form of a virtual source of information, data and ultimately knowledge, delivered through a web-based environment. A principal objective of DEKAS is to provide a gateway to accessing the right information and data at the right time within the overall process. To achieve this objective, identification of the information and data sources and flows between the various activities constituting the complete design process was required. In effect it was necessary to identify what, where and when data and information is utilized within the existing protection design and application process (Figure 1).

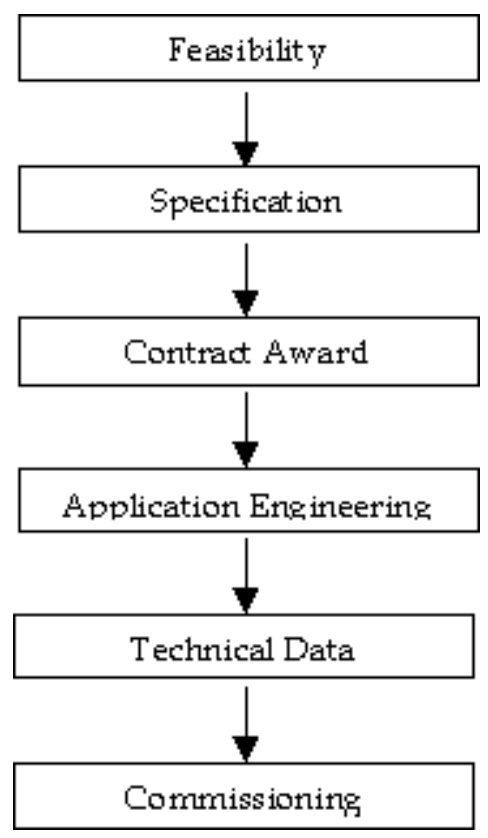

Figure 1: Generic protection design and application process model

This process knowledge was captured through the application of the KADS methodology (Wielinga et al., 1992), used to model the overall process on three inter-related layers, i.e. Task, Inference and Domain. A structured and progressive top-down approach, consisting of three phases, was adopted for the capture of the process knowledge:

- Phase 1 - Capture and representation of high-level knowledge relating to the main stages involved in the protection design and application process.

- Phase 2 - Capture and representation of detailed knowledge relating to the individual activities involved within each stage of the protection design and application process, and the identification of particular activities disposed to knowledge-based support.

- Phase 3-Capture and utilization of detailed activity knowledge required for the development of knowledge-based support for the activities identified in phase 2 . 


\section{HIGH-LEVEL REPRESENTATION OF THE GENERIC PROTECTION DESIGN AND APPLICATION PROCESS (PHASE 1)}

Initially, a high-level examination of the current protection design and application process was conducted independently on behalf of each company in conjunction with a nominated company expert. Once a high-level appreciation of the overall process had been gained, each company specific process was reviewed jointly with experts from both companies to identify areas of commonality. This resulted in the definition of a generic, six-stage, protection design and application process, representative of the high-level process implemented within each company. While the terminology used by both companies differed, the underlying activities, objectives and to some extent resources, were comparable.

\section{Description of the process stages}

Each stage of the generic design process is described below:

- The feasibility stage - This stage is concerned with the problem definition and development of possible solutions. During this stage, information is gathered on the existing system through site visits reports (and associated documentation). Following this, various high-level protection scheme options are proposed and assessed, resulting in an outline proposal being issued to the protection design engineer.

- The specification stage - This stage consists of the production of a technical specification detailing the required protection scheme functionality based on the outcomes received from the feasibility stage. During production of this specification, the major decisions relating to the protection scheme design are made. The specification is then circulated to prospective contractors.

- The contract award stage - During this stage the protection design engineer operates in an advisory capacity, providing a technical input to the assessment of the received tenders. Assessment of the suitability of each tender received from the various contractors is based on its compliance with the specification issued. Comparisons are made between each tender received in terms of the functionality offered by each contractor's proposed protection scheme design.

- The application engineering stage - This stage is concerned with the refinement of the contractor's proposed design and the resolution of contractor queries. During this stage the protection design engineer performs conformance checks on the contractor drawings and proposed equipment lists.

- The technical data stage - This stage is concerned with deriving the information required for the commissioning stage. Activities include performing thermal rating calculations and protection relay setting calculations, ensuring suitable coordination with interfacing protection schemes.

- The commissioning stage - This stage is concerned with bringing the newly installed protection scheme into service. During this stage the protection design engineer operates in a supportive role in association with the commissioning, where questions and problems arising during the commissioning process are resolved. 
The 'information roadmap'

The first stage in the development of DEKAS was the rapid development of a prototype system demonstrating the potential benefits of using web technology as a medium to display the captured knowledge. The high-level knowledge captured was represented in this early prototype system, forming an 'information roadmap'. This provided high-level access to relevant company-specific information and limited data resources for each generic stage of the defined process. Early representation of this high-level knowledge through the web-based system enabled the basic philosophy of DEKAS to be demonstrated. This exercise confirmed the web environment as an effective medium for the representation, company-wide dissemination and validation of the captured knowledge, and captured support for the continuing development of DEKAS.

Figure 2 illustrates a screenshot of the information roadmap system. On the left-hand side of the viewer is a list of menu options allowing access to various sources of information ranging from company documents; to the design process for each company; to background protection theory. The main window displays the captured knowledge, in this case part of the design process within the National Grid Company. In the process flow diagram each box is a link to more detailed information relating to that particular stage of the process.

\section{DETAILED REPRESENTATION OF THE PROTECTION DESIGN AND APPLICATION PROCESS (PHASE 2)}

Following the initial high-level examination of the protection design and application process, and the success of the 'information roadmap' prototype in confirming the webbased environment as the most suitable in which to develop DEKAS, the project focused on the capture of detailed process knowledge and the representation and implementation of this knowledge with in the DEKAS system. 


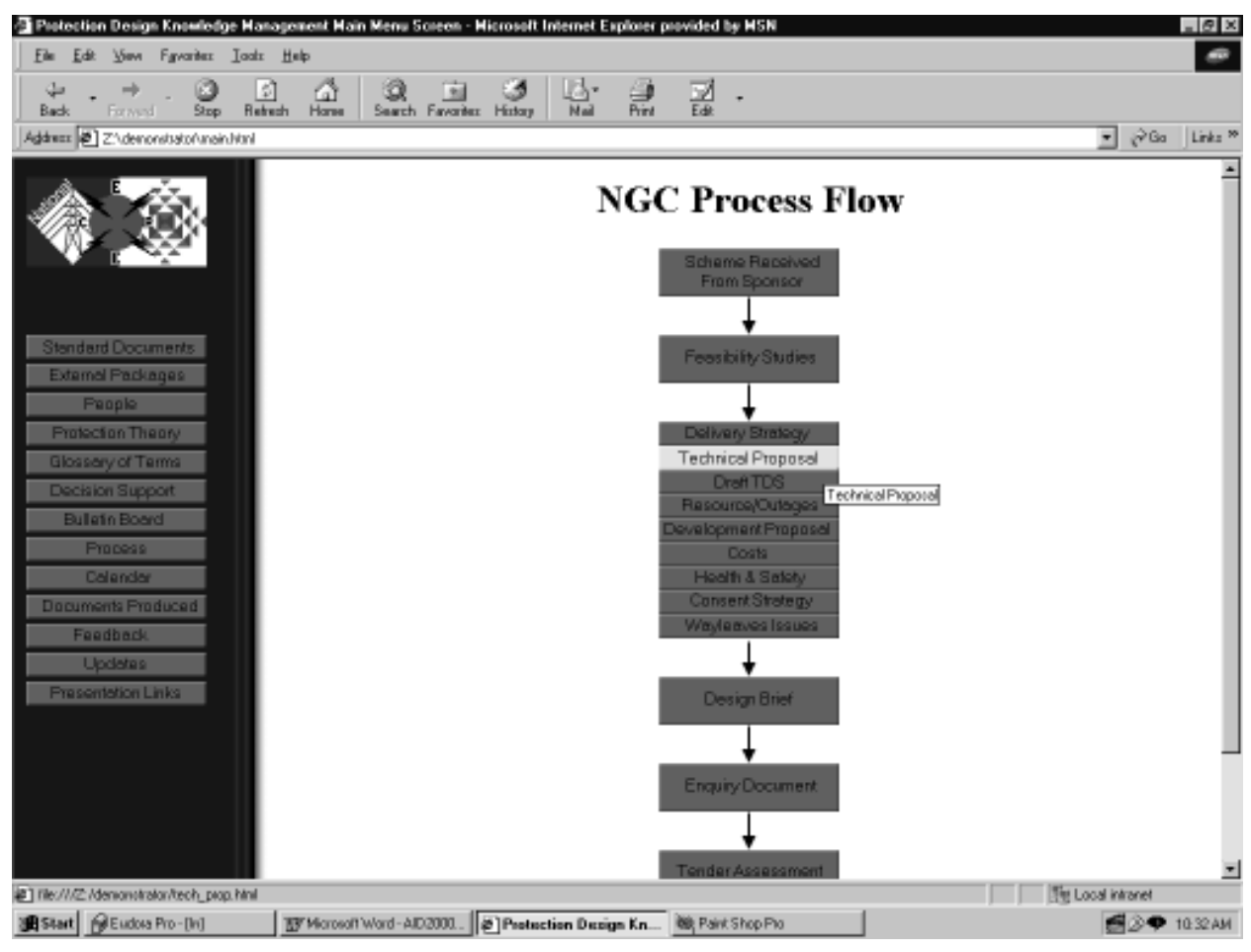

Figure 2 Screenshot of the information roadmap

Capture of detailed protection design and application process knowledge

The acquisition of company-specific process knowledge involved a series of face-to-face knowledge elicitation sessions conducted with representatives from each company. Typically, a session consisted of a structured discussion between one expert and two knowledge elicitors (Firlej and Hellens, 1991). The main objective of the knowledge elicitation sessions was to capture from a number of experts (i.e. design engineers), their specific role within the overall protection design and application process. Each design engineer had particular areas of expertise, therefore the role of each expert could be defined in terms of the particular activities in which they participated, ultimately resulting in a description of the overall process in terms of the activities involved and the data/information flows and interaction between each activity. Each knowledge elicitation session achieved this by:

- Identifying the activities with which each expert was primarily involved.

- Obtaining an overview of each activity identified.

- Identifying and describing the knowledge resources required to perform an activity. This knowledge may be tacit in nature (either known by the expert or by other individuals) or contained explicitly within documentation. This knowledge may be described in terms of:

- input data and information, e.g. project-specific documents, data, etc.

- references, e.g. general documents, policy statements, etc.

- tasks, i.e. sub-activities.

- Knowledge-based support (KBS), i.e. identification of activities particularly disposed to knowledge based support through the application of Artificial 
Intelligence (AI) techniques and consideration of the most appropriate form of KBS for each activity identified.

- outputs, e.g. documents, to databases, etc.

- Mapping each activity to the appropriate 'stage' of the generic protection design and application process flow (shown in Figure 1).

For each knowledge-elicitation session conducted it was essential to maintain a structured approach. For the capture of process knowledge a formalized tabular approach was adopted. The elicitors, effectively operating as facilitators, would guide the expert through the completion of standard tables used to describe each activity in terms if its inputs, references, tasks, KBS and outputs, as defined above. This maintained a focused approach to the knowledge elicitation process.

\section{Modelling of detailed protection design and application process}

Once the knowledge had been captured, it was formalized using the KADS modelling methodology which allows knowledge to be modeled on three interrelated layers:-

- Domain layer models

○ Represents the physical appreciation of Concepts, Properties, Relationships and Hierarchies of the problem domain.

- Task layer models

- Goal (describe purpose of task, local strategies).

- Method (describe subtasks to achieve goals).

- Inference layer models

- Describes the inputs and outputs associated with the identified tasks and their interaction within the reasoning process required to achieve a specific goal.

The tables utilized during the knowledge capture phase were developed with a view to mapping their contents across to a KADS model representation. 


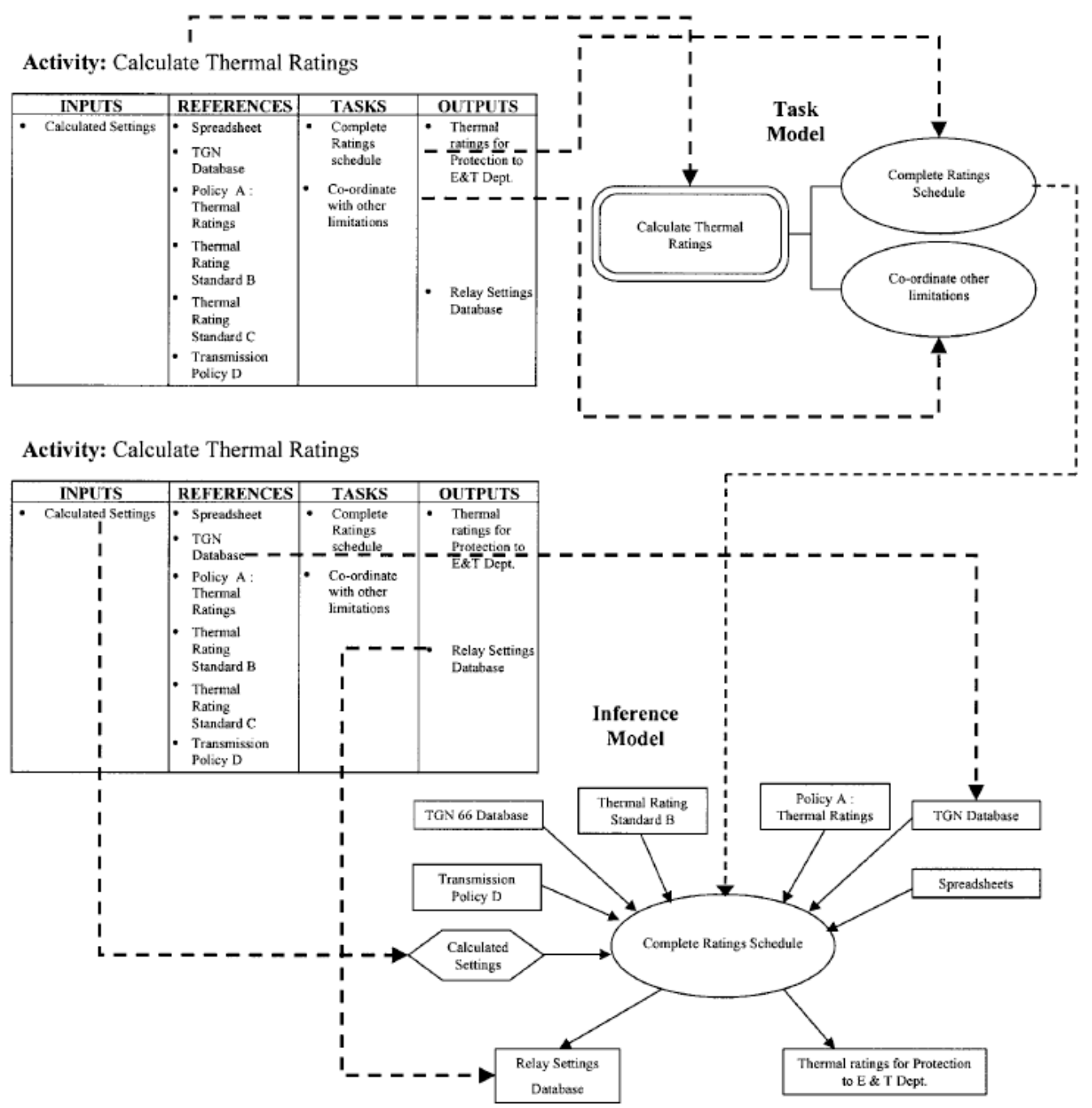

Figure 3: Mapping from table to task and inference models

This mapping process is illustrated in Figure 3. This shows the task model of 'Calculate Thermal Ratings' broken down into its two sub-tasks 'Complete Ratings Schedule' and 'Co-ordinate with Other Limitations'. The diagram also shows the inference model associated with the 'Complete Ratings Schedule' task illustrating the resources an engineer requires to complete the rating schedule. The outcome of this modelling phase of the project is a transparent graphical representation of the protection design and application process, which enabled:

- The identification of data/information sources and flows between the various activities constituting the design and application process. This will ultimately lead to the accessing of the right information and data at the right time within the context of a particular design project, i.e. what, where and when input and output information and data is utilized within the existing process.

- The identification of any shortcomings associated with the design process and resulting recommendations. 
- The identification of any supporting documentation required, which may not currently exist within the design process (e.g. guideline documents).

- The identification of future applications of knowledge-based support.

- A graphical representation of the outcomes from the knowledge-elicitation sessions, providing a useful platform for validation of the acquired process knowledge.

\section{Implementation of the overall protection design and application process}

The first-phase roll-out of the project consisted of the complete design process being represented graphically within the DEKAS web environment enabling the user (i.e. design engineers) to navigate throughout the modelled process as required. Figure 4 illustrates this navigation through the models within the web-browser. The top of the diagram shows a breakdown of the task and sub-tasks required to reach the inference model on the right. The lower half of the diagram represents the view the engineer is provided with. Starting from the left-hand side, the first rectangle shows a model of the highest level task and the four sub-tasks required to be completed.

Selecting one of these takes the user to a model of a breakdown of this sub-task. This process is repeated until the user reaches the inference model, which illustrates the resources required to complete the activity (shown in the rightmost rectangle in the diagram). Roll-out of DEKAS at this early stage of development benefited the further development of the system, despite no comprehensive document retrieval functionality existing (i.e. documents identified but no direct access through the DEKAS interface). Utilizing the capability of the web technology to create a widespread feedback forum enabled progressive validation of the system, offering prospective users the opportunity to comment on the accuracy of the modelled process and issues relating to the 'look and feel' of the system. This phased roll-out of the system gained the project exposure within the company and fostered a sense of ownership among the prospective users.

To give the design engineer physical access to the right information at the right time, as they progress through a protection design project, requires the integration of DEKAS with existing company repositories. The second-phase roll-out of the project involved a designated engineer linking the various resources identified in the models to their equivalent company repositories using a facility designed specifically for the purpose. 


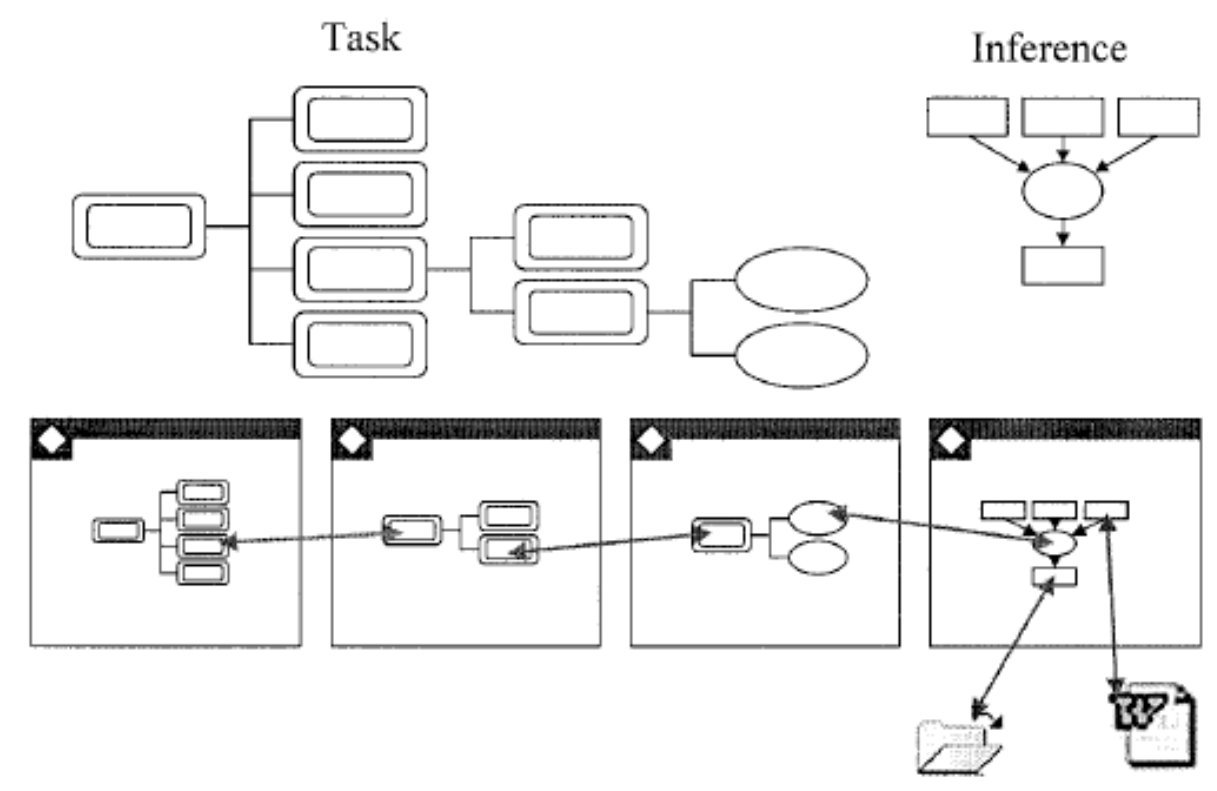

Figure 4: Representation of process through the DEKAS web environment

This achieved the main project objective of providing a gateway to a single 'virtual source' of information and data. The high-level architecture for this integration is illustrated in Figure 5. Success of the project will largely depend upon how much use is made of the final system on its introduction to each company. It is therefore important to encourage both companies to accept ownership of the final system and responsibility for its implementation and future maintenance. This issue has been addressed during the early stages of the development of DEKAS, where the system has been designed to ensure maintenance of the system will be a simple process for authorized company personnel. Each knowledge model from which the overall process is composed and associated information is stored in a database where system links are generated dynamically, eliminating the requirement for hard-coding of html script. Modifications or additions to the knowledge models within DEKAS can be affected by modifying the appropriate entry stored within the database. This is intended to safeguard against the system content (i.e. process details and documentation) becoming outdated, which may lead to a reduction in the reliability and use of the system, ultimately compromising the success of the project.

\section{FUTURE WORK (PHASE 3)}

Phase 1 and 2 of the project identified what, where and when information and data is utilized within the overall protection design and application process. The next phase of the project will focus on how better use can be derived from existing information and data resources to leverage knowledge as an important resource within each organization and provide support of the overall design and application process.

\section{Knowledge-based support}

Various process activities have been recognized as possible candidates for receiving knowledge-based support (KBS). The different forms of KBS under consideration are: 
- Automation of existing processes.

- Provision of checklists and circulation lists.

- Rule-based support derived from the capture of tacit knowledge relating to the decision- making and reasoning processes associated with specific activities.

- Case-based reasoning, enabling the retrieval of similar aspects of a design project.

Throughout the knowledge elicitation phase of the project the underlying approach taken by the majority of design engineers involved the re-use of knowledge relating to previous, similar design projects. The next stage of the project concentrates on emulating this intuitive reasoning process through the application of case-based reasoning techniques (Watson, 1997).

\section{Case-based Reasoning}

A prototype case-based reasoning (CBR) function is currently in development. It consists of a structured method of describing any protection design in terms of key characteristics. There is also an associated case library of previously completed protection designs to which the current protection design is compared. The closest match is returned to the user as a suggested design to examine. The engineer can then extract the relevant parts of the design and apply them to the current situation, re-using the experience and lessons learned from the previous design. Once the current design has been completed it is stored in the case library to be used in future, similar designs. This provides an effective resource of past experience which is available throughout the company. This means that an engineer need not be involved in a design to use the experience learned from it in the future.

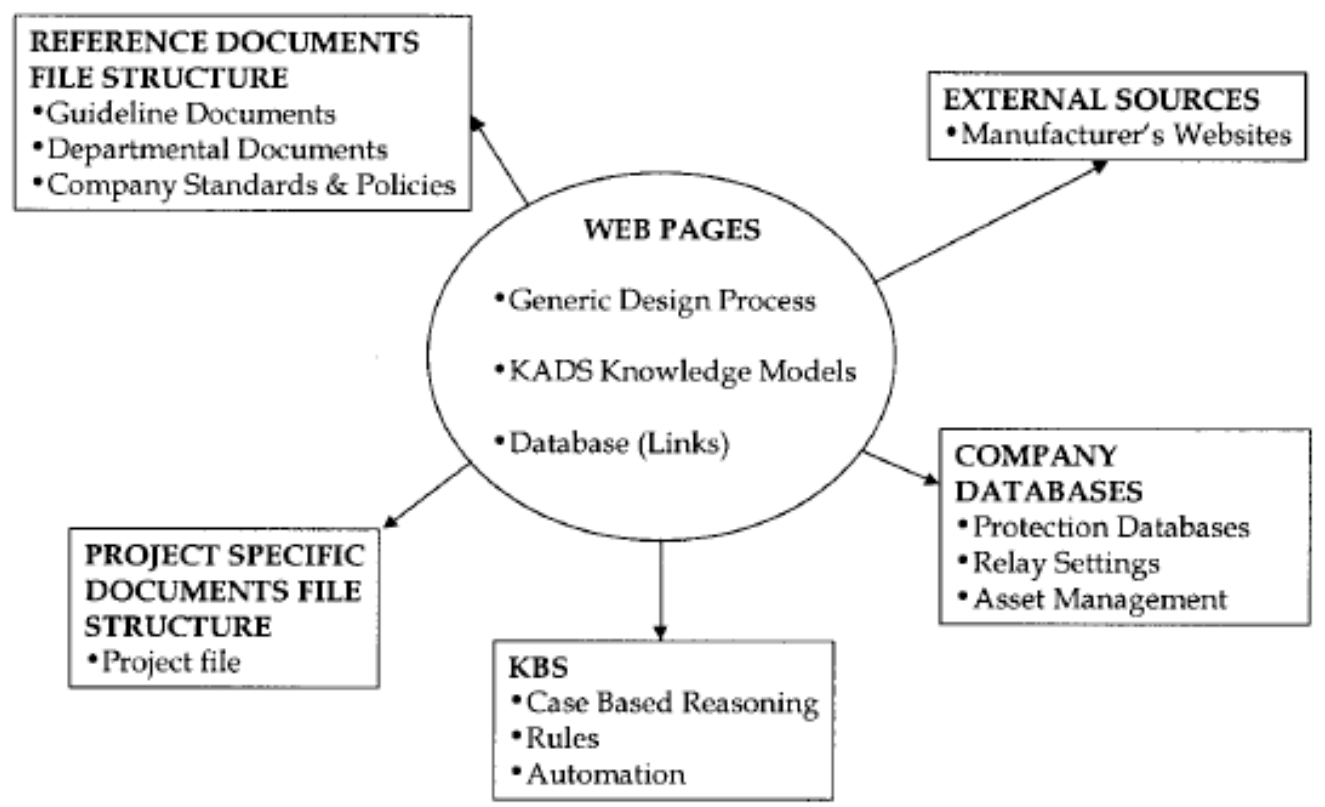

Figure 5: Architecture of DEKAS integration with existing company resources 
The development of DEKAS has been primarily involved in the domain of protection design. When part of the transmission grid requires upgrading or extending, several disciplines are involved, one of which is protection design. Other disciplines involved in this process include the layout of primary plant equipment and the design of the towers which carry the overhead lines. Similar to protection design, these areas also follow a process where a variety of resources are utilized by an engineer to solve a design problem and are being considered as target areas for applying the principles described in this paper. Indeed, there are often interfaces between the different disciplines at various stages of the project which would benefit from the development of similar systems. The techniques used in the development of DEKAS (i.e. modelled process, use of webtechnology, case-based reasoning) are not exclusive to protection design. Only the models themselves and the actual company repositories are specific to protection design. Therefore, the principles used in the development of DEKAS could equally be applied in different design domains, which also require the use of multiple knowledge resources to complete activities as part of a standard process. To utilize the case-based reasoning support, the designs or outcomes must be comparable to each other and should be able to be characterized in a standard case format.

\section{LESSONS LEARNED}

Through the development of DEKAS a number of lessons have been learned. The problems involved were generally concerned with cultural issues associated with getting people to use the system rather than issues relating to the technological aspects. The key lessons learned were:

- It is beneficial to get the involvement of the end-users from the outset. This allows the users to provide feedback on the ongoing development of the system, promoting a sense of ownership of the system. The phasing in of the system allows the user to become familiar with its operation over an extended period of time rather than being presented with the final system and being expected to use it straight away.

- It is important to stress to the engineer that the system is intended to support the current engineers design practice $\mathrm{s}$ rather than replacing them.

- To ensure the system is used, the end-user usually requires some immediate benefit to them. When phase 1 was rolled out (modeled process but no links to documentation) interest was limited. Following the incorporation of the links to documentation and supporting design file structure, the engineers were more receptive to its use.

\section{CONCLUSION}

The paper has presented the knowledge engineering approach taken to the acquisition and representation of existing process knowledge, and its contribution to the installment of a knowledge management culture within the electrical transmission system protection design and application domain. Implementation of a formalized, structured approach to the capture of process knowledge was adopted, facilitating the transition from the knowledge acquisition phase of the project to the knowledge representation phase using the KADS methodology. The use of web technology as an effective environment in which to represent, distribute and provide access to the captured knowledge has been 
demonstrated. The use of this technology as a vehicle for the all-important validation stage of the knowledge engineering process also proved invaluable in the progressive development of the DEKAS system. Future activity will concentrate on further design, development and implementation of knowledge-based support, in particular CBR, to enhance the management and utilization of data, information and knowledge within the protection design and application processes of both companies.

\section{ACKNOWLEDGEMENTS}

The authors gratefully acknowledge the contributions of ScottishPower and National Grid to the work described within this paper.

\section{REFERENCES}

Firlej M, Hellens D. 1991. Knowledge Elicitation —A Practical Handbook. Prentice-Hall International:London.

Schreiber Ahhermans, Anjewierden de Hoog Shadbolt,Van de Velde, Wielinga. 1999. Knowledge Engineering and Management. The MIT Press: Cambridge, MA.

Watson I. 1997. Applying case-based reasoning: techniques for enterprise systems. Morgan Kaufmann: San Mateo,CA.

Wielinga BJ, Schreiber A Th, Breuker JA. 1992. KADS: A modelling approach to knowledge engineering. Knowledge Acquisition 4.

Wright A, Christopoulos C. 1993. Electrical Power System Protection. Chapman and Hall: London. 